\title{
THE THEOREM OF THOMSON AND TAIT AND NATURAL
}

\section{FAMILIES OF TRAJECTORIES*}

BY

\section{EDWARD KASNER}

In most dynamical investigations relating to conservative forces, in particular those connected with the principle of least action and the developments of Hamilton and Jacobi, it is essential to group the possible motions according to the value of the constant $h$ representing the total energy. The trajectories corresponding to any given value of $h$ are said to form a natural $\dagger$ family. In the case of a particle moving in a three-dimensional conservative field, a natural family consists of $\infty^{4}$ curves. Examples are the $\infty^{4}$ straight lines of space, corresponding to zero force; and the $\infty^{4}$ vertical parabolas whose directrices are located in a fixed horizontal plane, corresponding to gravity assumed constant. In a paper published in the preceding volume of these Transactions, $\neq$ the general geometric character of natural families was expressed in terms of osculating circles; and a certain reciprocity, analogous to that of Scheffers for plane isogonal trajectories, was established.

In the present paper, which may be read independently, we start from the remarkable theorem due to Thomson and TAIT which states that the $\infty^{2}$ curves of a natural fanily which meet any surface orthogonally are necessarily orthogonal to $\infty^{1}$ surfaces, that is, form a normal congruence. Our main object is to show that this property belongs exclusively to natural fumilies. The new result may be regarded as a converse of Thomson and Tait's theorem and stated as follows :

If a quadruply-infinite system of curves in space is such that $\infty^{2}$ curves of the system meet an arbitrary surface orthogonally and always form a normal congruence (that is, admit $\infty^{1}$ orthogonal surfuces), then the system is of the natural type.

Natural families present themselves not only in the study of dynamical tra-

* Presented to the Society at the Princeton meeting, September 13, 1909.

†The term nutural was îrst used in this sense by PAInLevé, Journal de Mathématiques, vol. 10, 1894. Of course no connection is to be inferred with natural or intrinsic coördinates, that is, to invariance under the displacement group.

$\ddagger$ Natural families of trajectories: conservative fields of forces, these Transactions, vol. 10 (1909), pp. 201-219. This will be cited as earlier paper. 
jectories, but also, for example, in the discussion of brachistochrones, catenaries, and optical path curves. ${ }^{*}$ The many analogies that have been observed between these theories are based on the fact that they all lead to variation problems of the particular form $\dagger$

$$
\int F(x, y, z) d s=\text { minimum } \quad\left(d s=\sqrt{d x^{2}+d y^{2}+d z^{2}}\right),
$$

where $F$ is any point function and $d s$ is the element of length. From the purely mathematical point of view a natural family may be defined as the totality of extremals connected with such a variation problem.

In the kinetic interpretation $F$ represents the speed $v$, as found from the energy equation

$$
v^{2}=2(W+h),
$$

where $W$ denotes the work function (negative potential) and the mass is assumed to be unity. The integral above then represents the action, $\int v d s$, and the result is the principle of least action in the form given by Jacobi. The complete theorem of Thomson and Tait $\ddagger$ is as follows: "If from all points of an arbitrary surface particles not mutually influencing one another be projected normally with the proper velocities [so as to make the sum of the kinetic and potential energies have a given value]; points which they reach with equal actions lie on a surface cutting the paths at right angles." The $\infty^{1}$ orthogonal surfaces thus appear as surfaces of equal action. $\S$

In the optical interpretation, which is even more concrete, the function $F$ represents the index of refraction $\nu$, varying from point to point, and the integral $\int \nu d s$ is proportional to the time. We thus have Fermat's principle of least time. Thus the paths of light in an isotropic (not necessarily homogeneous) medium (for example, the atmosphere) form a natural family. Every natural family may be obtained in this way. The $\infty^{\prime}$ surfaces orthogonal to a congru. ence of paths present themselves as wave fronts (surfaces of equal time). $\|$

${ }^{*} \mathrm{Cf}$. A PPELL, Mécanique rationnelle, vol. 2 , p. 216. For oth.r interpretations see the introduction to the author's earlier paper.

† This form is characterized by the fact that the transversal relation reduces to orthogonality. The theorem of Thomson and Tait thus presents itself as a special case of Kneser's general theory of transversals. Cf. BoLzA, Variationsrechnung, pp. 131, 691.

$¥$ Trentise on natural philosophy (edition of 1879), part 1, section 332. Cf. DaRBoux, Théorie des suifaces, vol. 2, p. 468 ; A PPELL, Mécanique rationnelle, vol. 2 , p. 431.

\& A second converse which the author has obtained is as follows: If particles are projected normally from a base surface into a conservative field, and if the $\infty^{2}$ trajectories generated form a normal congruence, then the initial velocities must be such that the total energy is constant. This is true only "in general." In the case of central forces it is in fact possible to obtain normal congruences for which $h$ is not constant.

i) Usually the sets of wave surfaces so obtained will not form Lamé families, that is, cannot be regarded as members of triply orthogonal systems. The author has shown that the only natural families leading to Lamé families are those composed of circles; these oircles must be orthogonal to a tixed sphere (of real, imaginary, or zero radius). The corresponding optical and dynamical statements are yuite simple. 
In $\S 1$ the general condition that $\infty^{2}$ curves shall form a normal congruence is written out for the case of parametric representation. In our applications the curves are orthogonal to some base surface $\Sigma$ by assumption. It is therefore expedient to expand the general condition in powers of the parameter $t$ which, starting with the value zero for any point of $\Sigma$, varies along the orthogonal curve through that point. The result is then of the form

$$
\Omega_{1} t+\Omega_{2} t^{2}+\Omega_{3} t^{3}+\cdots=0 .
$$

For a normal congruence all the coefficients vanish. If the leading coefficient $\Omega_{1}$ vanishes the congruence may be described as "approximately of normal character." If the first $n$ coefficients vanish we describe the congruence as "approximately normal to the $n$th degree of approximation." A congruence orthogonal to a surface $\Sigma$ is approximately normal when and only when it is possible to construct $\infty^{2}$ curves osculating the given curves at the points of $\Sigma$ and forming a normal congruence (in the exact sense).

In $\S 2$ the converse theorem stated in italics above is proved. The result is then strengthened in two directions. In the first place it is sufficient to require that the $\infty^{2}$ curves, belonging to the quadruply-infinite system, which are orthogonal to an arbitrary surface $\Sigma$ shall form an approximately normal congruence. The discussion then shows that they necessarily form an exact normal congruence. In the second place it is sufficient to make this demand for suitable manifolds (of course infinite) of base surfaces, instead of for all surfaces.

Special cases of the theorem of Thomson and Tait arise where the selected base $\Sigma$ degenerates into a point, considered as a small sphere, or into a curve, considered as a thin tube. We show in $\S 4$ that the case of base points is not sufficient to ${ }^{\circ}$ characterize the natural type. Systems of $\infty^{4}$ curves exist, which, though not natural, have the property that the curves passing through an arbitrary point always form a normal congruence.

If for every curve as base the corresponding congruence is to be normal, the system is necessarily natural. If only the approximately normal character described above is demanded, the more general class of velocity systems is obtained $(\S 3)$. This type was defined in our earlier paper in terms of motion (loci of equal speed) in connection with an arbitrary field of force.* Only for conservative fields are the velocity systems of the natural type. The general velocity system is represented by equations of the form

$$
y^{\prime \prime}=\left(\psi-y^{\prime} \phi\right)\left(1+y^{\prime 2}+z^{\prime 2}\right), \quad z^{\prime \prime}=\left(\chi-z^{\prime} \phi\right)\left(1+y^{\prime 2}+z^{\prime 2}\right),
$$

where $\phi, \psi$, and $\chi$ are arbitrary point functions, and is characterized by the property that the osculating circles constructed at any point $p$ for the $\infty^{2}$ curves

* These 'Transactions, vol. 10 (1909), p. 376. 
passing through that point meet at another point $P$, thus forming a bundle (property $A$ ).*

Natural families are distinguished from other velocity systems by an additional peculiarity, property $B$, or its equivalent $D$, of our earlier paper. $\dagger$ In $\S 4$ of the present paper it is shown that this is simply expressed by the requirement that the curves through any point form a normal congruence. Thus the natural type is characterized by these two properties: the curves through any point admit $\infty^{1}$ orthogonal surfaces, and the osculating circles constructed at the common point form a bundle.

In the last section $(\S 5)$ a contribution is made to the general theory of quadruply-infinite systems of curves in space, the only assumption being that $\infty^{2}$ curves pass through every point, one in each direction. It is shown that with any system there are associated certain surfaces which when employed as bases give rise to congruences of approximately normal character..$_{+}^{+}$These surfaces satisfy a certain partial differential equation of the second order. In the particular case of the velocity type this equation reduces to one of the first order, and in the still more special case of the natural type it vanishes identically.

Although the discussion of the present paper is restricted to ordinary space of three dimensions, the results are, in all probability, valid for higher spaces also. In the case of two dimensions, however, the geometric part of Thomson and Tait's theorem becomes trivial since any system of $\infty^{1}$ curves necessarily admits orthogonal curves; the converse proposition is then not true.

\section{§1. Conditions for an exaCt or approximate nORMal CONGRUence.}

The condition that a congruence of curves, represented in the usual form by

$$
\frac{d x}{A(x, y, z)}=\underset{B(x, y, z)}{d y}=\frac{d z}{C(x, y, z)},
$$

shall be normal is that the corresponding total equation

$$
A d x+B d y+C d z=0
$$

shall be exact; this gives the familiar result

$$
A\left(B_{z}-C_{y}\right)+B\left(C_{x}-A_{z}\right)+C\left(A_{y}-B_{. x}\right)=0 .
$$

* The analogous systems in the plane, $y^{\prime \prime}=\left(\psi-y^{\prime} \phi\right)\left(1+{y^{\prime}}^{2}\right)$, were first studied by Scheffers, Leipziger Berich te (1898), in connection with isogonal trajectories.

† Property $B$ states the mutual orthogonality of the three hyperosculating circles existing in any bundle. Property $D$ may be expressed in simplified form as follows: The transformation $T$ from the point $p$ to the point $P$ is such that the three lineal elements at $p$ each of which is converted by $T$ into a cocircular element at $P$ are mutually orthogonal.

$\ddagger$ In general no exactly normal congruences can be obtained.

$\S$ The results of our earlier paper hold for all dimensions. That the present results remain valid in three-dimensional spaces of constant curvature may be shown by conformal representation. 
A congruence of curves defines a field of $\infty^{3}$ direction elements in space. If these elements are given in terms of three arbitrary parameters $u, v, w$ (instead of $x, y, z)$ the field takes the form

$$
\begin{gathered}
x=X(u, v, w), \quad y=Y(u, v, w), \quad z=Z(u, v, w), \\
d x: d y: d z=\bar{X}(u, v, w): \bar{Y}(u, v, w): \bar{Z}(u, v, w) .
\end{gathered}
$$

The condition for a normal congruence then becomes

$$
\bar{X}\{(\bar{Y} X Y)-(\bar{Z} Z X)\}+\bar{Y}\{(\bar{Z} Y Z)-(\bar{X} X Y)\}+\bar{Z}\{(\bar{X} Z X)-(\bar{Y} Y Z)\}=0 \text {, }
$$

where the parentheses denote jacobians of the enclosed functions with respect to the parameters, so that, for example,

$$
(\bar{Y} X Y) \equiv\left|\begin{array}{ccc}
\bar{Y}_{u} & \bar{Y}_{v} & \bar{Y}_{v} \\
X_{u} & X_{v} & X_{v} \\
Y_{u} & Y_{v} & Y_{w}
\end{array}\right| .
$$

In the applications below our congruence admits one orthogonal surface $\Sigma$ by assumption, and we may take our parameters relative to it as follows: Let $u$, $v$ denote Gaussian parameters on $\Sigma$, and let $w$ be the parameter which varies as the point describes one of the orthogonal curves, assuming it to be zero as the point starts out from the surface. (In particular $w$ might represent the distance from $\Sigma$ along the curve.) The congruence then takes the form

$$
\begin{aligned}
& X=x+A_{1} w+\frac{1}{2} A_{2} w^{2}+\cdots, \\
& Y=y+B_{1} w+\frac{1}{2} B_{2} w^{2}+\cdots, \\
& Z=z+C_{1} w+\frac{1}{2} C_{2} w^{2}+\cdots, \\
& \bar{X}=A_{1}+A_{2} w+\frac{1}{2} A_{3} w^{2}+\cdots, \\
& \bar{Y}=B_{1}+B_{2} w+\frac{1}{2} B_{3} w^{2}+\cdots, \\
& \bar{Z}=C_{1}+C_{2} w+\frac{1}{2} C_{3} w^{2}+\cdots,
\end{aligned}
$$

the last three functions being found by differentiating the first three with respect to $w$. Here the coefficients are all functions of $u$ and $v$; for $x, y, z$ are to be replaced by the functions defining the surface $\Sigma ; A_{1}, B_{1}, C_{1}$ are proportional to the direction cosines of the normal to the surface; and the remaining coefficients are arbitrary.

The condition for a normal congruence, expanded in powers of $w$, now takes the form

$$
\Omega_{1} w+\Omega_{2} w^{2}+\Omega_{3} w^{3}+\cdots=0,
$$

where $\Omega_{i}$ depends for its value upon the quantities $A_{1}, B_{1}, C_{1}, \ldots, A_{i+1}, B_{i+1}, C_{i+1}$, involving only partial derivatives of the first order. The absolute term neces- 
sarily vanishes, since the curves are orthogonal to $\Sigma$ for which $w=0$ by assumption.

In case the congruence is normal all the coefficients $\Omega$ will vanish. If the first coefficient $\Omega_{1}$ vanishes then the congruence may be said to be normal to the first degree of approximation. In this case the curves may be regarded as orthogonal to some surface infinitesimally close to $\Sigma$. In general the congruence will be said to be normal to the $n$th degree of approximation (or to terms of the $n$th order) provided

$$
\Omega_{1}=\Omega_{2}=\cdots=\Omega_{n}=0 .
$$

In the following applications we take up first the general case where $\Sigma$ is a proper surface, and then the special cases where $\Sigma$ reduces to a curve and to a point respectively. In the case of surface and curve we shall make use of the explicit value of $\Omega_{1}$ only; in the case of a point it will appear that $\Omega_{1}$ vanishes identically and it will be necessary to calculate $\Omega_{2}$ and $\Omega_{3}$.

\section{§ 2. General case where the base is a proper surface.}

Consider an arbitrary quadruply infinite system of curves in space, assuming that one passes through each point in each direction. Such a system may be defined by a pair of differential equations of the second order

$$
y^{\prime \prime}=F\left(x, y, z, y^{\prime}, z^{\prime}\right), \quad z^{\prime \prime}=G\left(x, y, z, y^{\prime}, z^{\prime}\right),
$$

where $F$ and $G$ are uniform functions which we assume to be analytic in the five arguments. Denoting the initial values of $x, y, z, y^{\prime}, z^{\prime}$, which may be taken at random, by $x, y, z, p, q$ respectively, and employing $X, Y, Z$ as current coördinates, we may write the solutions of (1) in the form

$$
\begin{aligned}
& Y=y+p(X-x)+\frac{1}{2} F(X-x)^{2}+\frac{1}{6} M(X-x)^{3}+\cdots, \\
& Z=z+q(X-x)+\frac{1}{2} G(X-x)^{2}+\frac{1}{6} N(X-x)^{3}+\cdots
\end{aligned}
$$

Here $F$ and $G$ are expressed as functions of $x, y, z, p, q$, and $M$ and $N$, found by differentiating $(1)$, are given by

$$
\begin{gathered}
M \equiv F_{x}+p F_{y}+q F_{z}+F F_{p}+G F_{q}, \\
N \equiv G_{x}+p G_{y}+q G_{z}+F G_{p}+G G_{q} .
\end{gathered}
$$

The terms of higher order will not be needed in our discussion. Equations (2) involve five arbitrary parameters but of course represent only $\propto^{4}$ curves.

Consider now an arbitrary surface $\Sigma$

$$
z=f(x, y) \text {. }
$$


At each point of this surface and normal to it a definite curve of the given family (1) may be constructed. A certain congruence will thus be determined. We wish to express the condition that this shall be of the normal type, that is, that the $\infty^{2}$ curves shall admit a family of orthogonal surfaces.

The direction normal to the surface $\Sigma$ at any point is given by

so that

$$
1: p: q=f_{x}: f_{y}:-1,
$$

$$
p=P(x, y), \quad q=Q(x, y),
$$

where

$$
P \equiv \frac{f_{y}}{f_{x}}, \quad Q \equiv-\frac{1}{f_{x}} .
$$

These functions are connected by the relation

$$
P Q_{x}-Q P_{x}-Q_{y} \equiv 0 .
$$

The equations of the $\infty^{2}$ curves corresponding to the given initial conditions may now be written

$$
\begin{aligned}
& Y=y+P t+\frac{1}{2} \bar{F} t^{2}+\frac{1}{6} \bar{M} t^{3}+\cdots, \\
& Z=f+Q t+\frac{1}{2} \bar{G} t^{2}+\frac{1}{6} \bar{N} t^{3}+\cdots,
\end{aligned}
$$

where $t$ takes the place of $X-x$ in (2), and where the bars indicate that the substitution (4), (5) has been carried out, so that, for example,

$$
\bar{F}(x, y) \equiv F(x, y, f, P, Q) .
$$

The coefficients of the powers of $t$ in (6) are thus functions of the two parameters $x, y$.

The congruence (6) defines a field of lineal elements, which, in terms of the three parameters $t, x, y$, is given by

$$
\begin{aligned}
& X=x+t, \\
& Y=y+P t+\frac{1}{2} \bar{F} t^{2}+\frac{1}{6} \bar{M} t^{3}+\cdots, \\
& Z=f+Q t+\frac{1}{2} \bar{G} t^{2}+\frac{1}{6} \bar{N} t^{3}+\cdots, \\
& Y^{\prime}=P+\bar{F} t+\frac{1}{2} \bar{M} t^{2}+\cdots, \\
& Z^{\prime}=Q+\bar{G} t+\frac{1}{2} \bar{N} t^{2}+\cdots
\end{aligned}
$$

The condition for a normal congruence is *

$$
\left(I^{\prime} X Y\right)-\left(Z^{\prime} Z X\right)+Y^{\prime}\left(Z^{\prime} Y Z\right)-Z^{\prime}\left(Y^{\prime} Y Z\right)=0,
$$

* In the present case $\bar{X}: \bar{Y}: \bar{Z}=1: Y^{\prime}: Z^{\prime}$. We may also use the convenient form due to Beltrami. Cf. BianchI-LUkat, Differentialgeometrie, p. 340. 
where the parentheses denote jacobians taken with respect to $t, x, y$. For example:

$$
\left(Y^{\prime} X Y\right)=\left|\begin{array}{ccc}
\bar{F}+\bar{M} t+\cdots & P_{x}+\bar{F}_{x} t+\cdots & P_{y}+\bar{F}_{y} t+\cdots \\
1 & 1 & 0 \\
P+\bar{F} t+\cdots & P_{x} t+\cdots & 1+P_{y} t+\cdots
\end{array}\right|
$$

Expanding our results in powers of $t$, and writing down only the terms of first degree, we find

$$
\begin{aligned}
& \left(Y^{\prime} X Y\right)=\bar{F}-P P_{y}-P_{x}+t\left\{\bar{M}-\bar{F}_{x}-P \bar{F}_{y}\right\}+\cdots, \\
& \left(Z^{\prime} Z X\right)=Q_{x} f_{y}-Q_{y} f_{x}+Q Q_{y}-G f_{y}+t\left\{-\bar{N} f_{y}+\bar{G}_{x} f_{y}-\bar{G}_{y} f_{x}+Q \bar{G}_{y}\right\}+\cdots, \\
& \left(Z^{\prime} Y Z\right)=Q Q_{x}+P\left(Q_{y} f_{x}-Q_{x} f_{y}\right)-\bar{G} f_{x}+t\left\{-\bar{N} f_{x}+\bar{F}\left(Q_{y} f_{x}-Q_{x} f_{y}\right)\right. \\
& \left.+\bar{G}\left(P_{x} f_{y}-P_{y} f_{x}\right)+Q G_{x}+P\left(G_{y} f_{x}-G_{x} f_{y}\right)+Q\left(Q_{x} P_{y}-P_{x} Q_{y}\right)\right\}+\cdots, \\
& \left(Y^{\prime} Y Z\right)=Q P_{x}+P P_{y} f_{x}-P P_{x} f_{y}-\bar{F} f_{x} \\
& +t\left\{-\bar{M} f_{x}+Q \bar{F}_{x}-\bar{F} Q_{x}+\bar{G} P_{x}+P\left(\bar{F}_{y} f_{x}-\bar{F}_{x} f_{y}+Q_{x} P_{y}-P_{x} Q_{y}\right)\right\}+\cdots
\end{aligned}
$$

Substituting these values in (8), we find that the part free from $t$ is

$$
\frac{1}{Q}\left(1+P^{2}+Q^{2}\right)\left(P Q_{x}-Q P_{x}-Q_{y}\right)
$$

which vanishes in consequence of $\left(5^{\prime \prime}\right)$. This is as it should be, since our $\infty^{2}$ curves are orthogonal to $\Sigma$ by construction.

The terms containing the first power of $t$ give

$$
\begin{aligned}
\left(1+P^{2}\right. & \left.+Q^{2}\right)\left(P \bar{G}_{x}-Q \bar{F}_{x}-\bar{G}_{y}\right) \\
& +2 \bar{F}\left\{\left(P^{2}+Q^{2}\right)\left(Q_{x}-P Q_{y}\right)+2 \bar{G} f P P_{y}-\left(P^{2}+Q^{2}\right) P_{x}\right\}=0 .
\end{aligned}
$$

From $\left(6^{\prime}\right)$ we find

$$
\bar{F}_{x}=F_{x}+F_{z} f_{x}+F_{p} P_{x}+F_{q} Q_{x},
$$

with corresponding results for $\bar{G}_{x}$ and $\bar{G}_{y}$. Substituting these values, and observing from (5) and $\left(5^{\prime}\right)$ that

$$
f_{x}=-\frac{1}{Q}, \quad f_{y}=-\frac{P}{Q}, \quad Q_{y}=P Q_{x}-Q P_{x},
$$

we may reduce $(10)$ to

$$
\begin{aligned}
& 2 F\left\{P Q P_{x}+Q^{2} Q_{x}\right\}+2 G\left\{P P_{y}-\left(P^{2}+Q^{2}\right) P_{x}\right\}-\left(1+P^{2}+Q^{2}\right) \times \\
& \left\{Q F_{x}-F_{z}-P G_{x}+G_{y}+\left(Q F_{p}-Q G_{q}-P G_{p}\right) P_{x}+G_{p} P_{y}+Q F_{q} Q_{x}\right\}=0 .
\end{aligned}
$$


This is then a necessary condition in order that the $\infty^{2}$ curves belonging to the quadruply infinite system (1) and orthogonal to the surface (4) shall form a normal congruence. The result is to hold in virtue of (4) and (5).

It is of course not a sufficient condition. It merely expresses the fact that the curves orthogonal to $\Sigma$ are also orthogonal to some consecutive surface, that is, that the congruence is approximately normal to the first degree.

Our main problem is to find all systems (1) which have the orthogonality property with respect to every base surface $\Sigma$. It is then necessary that $\left(10^{\prime}\right)$ should be true for an arbitrary function $f(x, y)$. The function can be so selected that for any chosen values of $x$ and $y$ the quantities, $f, P, Q, P_{x}$, $P_{y}, Q_{x}$, shall take on arbitrary numerical values; for the only relation to be fulfilled is $\left(5^{\prime \prime}\right)$ and this merely determines $P_{y}$. The condition $\left(10^{\prime}\right)$ must therefore hold identically. Arranging it in the form

$$
\left(1+P^{2}+Q^{2}\right) C_{0}+Q C_{1} Q_{x}+C_{2} P_{y}-C_{3} P_{x}=0,
$$

and equating coefficients to zero, we find

$$
\begin{aligned}
& C_{0} \equiv q F_{x}-F_{z}-p G_{x}+G_{y}=0, \\
& C_{1} \equiv\left(1+p^{2}+q^{2}\right) F_{q}-2 q F=0, \\
& C_{2} \equiv\left(1+p^{2}+q^{2}\right) G_{p}-2 p G=0, \\
& C_{3} \equiv\left(1+p^{2}+q^{2}\right)\left\{q G_{q}+p G_{p}-q F_{p}\right\}+2 p q F-2\left(p^{2}+q^{2}\right) G=0 .
\end{aligned}
$$

Integration of the second and third of these partial differential equations gives

$$
F=f_{1}(p, x, y, z)\left(1+p^{2}+q^{2}\right), \quad G=g_{1}(q, x, y, z)\left(1+p^{2}+q^{2}\right),
$$

where $f_{1}$ and $g_{1}$ denote unknown functions of the four arguments indicated. Substituting these values in the fourth equation, we find

and therefore

$$
f_{1 p}=g_{1 q},
$$

$$
f_{1}^{\prime}=\psi-p \phi, \quad g_{1}=\chi-q \phi,
$$

where $\phi, \psi, \chi$ are functions of $x, y, z$ only. The general solution of the last three equations of the set (11) is therefore

$$
F=(\psi-p \phi)\left(1+p^{2}+q^{2}\right), \quad G=(\chi-q \phi)\left(1+p^{2}+q^{2}\right) .
$$

We have still to satisfy the first equation of $(11)$, which now reduces to

$$
\psi_{z}-\chi_{y}+p\left(\chi_{x}-\phi_{z}\right)+q\left(\phi_{y}-\psi_{x}\right)=0 .
$$


The functions $\phi, \psi, \chi$ must therefore satisfy the equations

$$
\psi_{z}-\chi_{y}=0, \quad \chi_{x}-\phi_{z}=0, \quad \phi_{y}-\psi_{x}=0,
$$

and hence are expressible as the derivatives of a common function in the form

$$
\phi=L_{x}, \quad \psi=L_{y}, \quad \chi=L_{z} .
$$

The solutions of the set (11) are therefore

$$
F=\left(L_{y}-p L_{x}\right)\left(1+p^{2}+q^{2}\right), \quad G=\left(L_{z}-p L_{x}\right)\left(1+p^{2}+q^{2}\right),
$$

involving an arbitrary function $L$ of $x, y, z$. The resulting system (1) is thus recognized to be a natural family.* This gives our fundamental

Theorem I. If a quadruply infinite system of curves in space, one passing in each direction through each point, has the property that those $\infty^{2}$ members of it which are orthogonal to an arbitrarily selected surface always form a normal congruence (that is, admit $\infty^{1}$ orthogonal surfaces), then the system must be a natural family.

That natural families of trajectories actually have this property is known from Thomson and Tait's theorem. In the above discussion use has been made not of the complete condition for a normal congruence, but only of condition $\left(10^{\prime}\right)$ derived from the terms of the first order in $t$. We may therefore state a stronger converse result as follows :

Theorem II. The only systems of $\infty^{4}$ curves which have the mroperty that the curves orthogonal to any surface are always orthogonal to sor ; infinitesimally neighboring surface are those of the natural type.

If a congruence of curves meets two neighboring surfaces orthogonally it need not meet $\infty^{1}$ surfaces orthogonally and therefore it approximates but need not coincide with a normal congruence. The above theorem shows however that if the weak requirement of approximate normal character be imposed on all the congruences obtained from the given quadruply infinite system, they will all be exactly normal.

We may further strengthen our theorem by demanding the orthogonality property for some instead of all surfaces. Our fundamental equations (11) resulted from the fact that $x, y, z, f, P, Q, P_{x}, P_{y}, Q_{x}$, might receive arbitrary numerical values. It will therefore be sufficient to take a manifold of surfaces sufficiently large to leave these quantities, or the equivalent quantities

$$
x, y, z, z_{x}, z_{y}, z_{x x}, z_{x y}, z_{y y},
$$

unrestricted. Since these quantities define a differential surface element of the second order, we may state the result as follows :

* See these Transactions, vol. 10 (1909), p. 204. 
THeOREM III. If in theorem II, and, a fortiori, in theorem 1 , we consider instead of all surfaces, a manifold of surfaces containing all the $\infty^{8}$ differential elements of second order (15), the results are still valid.

The smallest manifolds of this sort are composed of $\infty^{6}$ surfaces. The totality of quadrics would be more than sufficient. The paraboloids

$$
z=a+b x+c y+a_{1} x^{2}+b_{1} x y+c_{1} y^{2}
$$

would be sufficient. In the discussion of base curves and points that follows, smaller manifolds of bases will present themselves.

\section{Case Where the base is a curve.}

We now consider those curves of a given system

$$
y^{\prime \prime}=F\left(x, y, z, y^{\prime}, z^{\prime}\right), \quad z^{\prime \prime}=G\left(x, y, z, y^{\prime}, z^{\prime}\right)
$$

which meet an arbitrary curve

$$
y=f(x), \quad z=g(x)
$$

orthogonally, and impose the condition for a normal congruence (to first approximation).

The $\infty^{2}$ possible initial elements $(x, y, z, p, q)$ may be written in terms of two parameters $x$ and $p$ as follows :

where

$$
y=f(x), \quad z=g(x), \quad q=R p+S,
$$

$$
R \equiv-\frac{f^{\prime}}{g^{\prime}}, \quad S \equiv-\frac{1}{g^{\prime}}
$$

This results from the orthogonality of the directions $(1: p: q)$ and $\left(1: f^{\prime}: g^{\prime}\right)$, expressed by

and giving

$$
\begin{gathered}
1+p f^{\prime}+q g^{\prime}=0, \\
q=S+p R .
\end{gathered}
$$

The congruence orthogonal to the base curve (16) may be written as a direction field in the form

$$
\begin{aligned}
X & =x+t, \\
Y & =f+p t+\frac{1}{2} \bar{F} t^{2}+\cdots, \\
Z & =g+q t+\frac{1}{2} \bar{G} t^{2}+\cdots, \\
Y^{\prime} & =p+\bar{F} t+\frac{1}{2} \bar{M} t^{2}+\cdots, \\
Z^{\prime} & =S+p R+\bar{G} t+\frac{1}{2} \bar{N} t^{2}+\cdots,
\end{aligned}
$$


where the parameters are $t, x$, and $p$. The bars indicate that the substitution (16), (18) has been effected, so that, for example,

$$
\bar{F}(x, p)=F(x, f, g, p, S+p R) .
$$

The requisite jacobians taken with respect to $t, x, p$ are

$$
\begin{aligned}
& \left(Y^{\prime} X Y\right)=f^{\prime}-p+t\left(f^{\prime}-p\right) \bar{F}_{p}+\cdots, \\
& \left(Z^{\prime} Z X\right)=R\left(S+p R-g^{\prime}\right)+t\left(S+p R-g^{\prime}\right) \bar{G}_{p}+\cdots, \\
& \left(Z^{\prime} Y Z\right)=R\left\{p g^{\prime}-f^{\prime}(S+p R)\right\}+t\left[(S+p R)\left(S^{\prime}+p R^{\prime}\right)\right. \\
& \left.+g R \bar{F}-g^{\prime} \bar{G}+\left\{p g^{\prime}-f^{\prime}(S+p R)\right\} \bar{G}_{p}\right]+\cdots, \\
& \left(Y^{\prime} Y Z\right)=p g^{\prime}-f^{\prime}(S+p R)+t\left[p\left(S^{\prime}+p R^{\prime}\right)\right. \\
& \left.+f^{\prime} R \bar{F}-f^{\prime} \bar{G}+\left\{p g^{\prime}-f^{\prime}(S+p R)\right\} \bar{F}_{p}\right]+\cdots
\end{aligned}
$$

Developing the condition for a normal congruence in powers of $t$, we find that the absolute term vanishes identically, while the coefficient $\Omega_{1}$ of the first power gives

$$
\begin{aligned}
& (R-p S) \bar{F}_{p}-\{1+p S(S+p R)\} G_{p}-2 R^{2}(S+p R) \bar{F}+p G-2 R \bar{F} \\
& \quad+R(S+p R) \bar{G}-\{p+R(S+p R)\}\left\{p \bar{G}_{p}-(S+p R) \bar{F}_{p}-G\right\}=0 .
\end{aligned}
$$

Eliminating $S$ by means of (18), and substituting the values

$$
\bar{F}_{p}=F_{p}+R F_{q}, \quad \bar{G}_{p}=G_{p}+R G_{q},
$$

derived from $\left(19^{\prime}\right)$, we may reduce $(20)$ to

$$
\begin{aligned}
2_{p} G=\left(1+p^{2}+q^{2}\right) G_{p}+R\left[\left(1+p^{2}\right.\right. & \left.\left.+q^{2}\right)\left(F_{p}-G_{q}\right)-2 p F+2 q G\right] \\
& +R^{2}\left[\left(1+p^{2}+q^{2}\right) F_{q}-2 q F\right]=0 .
\end{aligned}
$$

If this is to hold for all curves (16) it must be an identity. For the curve (16) can be so selected that $x, y, z, p, q, R$ receive arbitrarily assigned numerical values. Hence we have the three partial differential equations

$$
\begin{aligned}
\left(1+p^{2}+q^{2}\right) F_{q}-2 q F & =0, \\
\left(1+p^{2}+q^{2}\right) G_{\dot{p}}-2 p G & =0, \\
\left(1+p^{2}+q^{2}\right)\left(F_{p}-G_{q}\right)-2 p F+2 q G & =0 .
\end{aligned}
$$

This set is seen to be equivalent to the last three of the equations (11). The general solution is

$$
F=(\psi-p \phi)\left(1+p^{2}+q^{2}\right), \quad G=(\chi-p \phi)\left(1+p^{2}+q^{2}\right),
$$


involving three arbitrary functions of $x, y, z$. The quadruply infinite system (1) thus takes the form

$$
y^{\prime \prime}=\left(\Psi-y^{\prime} \phi\right)\left(1+y^{\prime 2}+z^{\prime 2}\right), \quad z^{\prime \prime}=\left(\chi-z^{\prime} \phi\right)\left(1+y^{\prime 2}+z^{\prime 2}\right) .
$$

This is recognized to be a velocity system, according to the terminology of the author's earlier paper. It is characterized geometrically by the property that the circles of curvature constructed at any point for those curves of the system which pass through that point form a bundle, i. e., have another point in common (property $A$ ). We have made use of merely one term in the complete condition for a normal congruence. Hence our result is

THeOREM IV. Every velocity system has the property that those members of it which meet an arbitrarily selected base curve* orthogonally are also orthogonal to some neighboring surface (necessarily tubular). Conversely, if a quadruply infinite system has this property for all base curves it will be of the velocity type.

The converse result remains valid if instead of all base curves we take a manifold which allows $x, f, g, f^{\prime}, g^{\prime}$ to take arbitrary values. The smallest number of base curves that would be sufficient is $\infty^{4}$. Two adequate manifolds of curves are made use of in the following

THEOREM V. The converse result of theorem IV remains valid if the only bases employed are the $\infty^{4}$ straight lines of space; or, the $\infty^{4}$ curves of the system itself.

\section{§4. Case where the base is a point.}

When the base is a point orthogonality means merely incidence. Consider then the $\infty^{2}$ curves of the system (1) which pass through a given point $(x, y, z)$. Define the initial direction by $y^{\prime}=p, z^{\prime}=q$, where $p$ and $q$ are now arbitrary; the resulting congruence takes the form

$$
\begin{aligned}
& X=x+t \\
& Y=y+p t+\frac{1}{2} F t^{2}+\frac{1}{6} M t^{3}+\cdots \\
& Z=z+q t+\frac{1}{2} G t^{2}+\frac{1}{6} N t^{3}+\cdots \\
& Y^{\prime}=p+F t+\frac{1}{2} M t^{2}+\cdots \\
& Z^{\prime}=q+G t+\frac{1}{2} N t^{2}+\cdots
\end{aligned}
$$

Our jacobians, calculated to terms of the third order in $t$, instead of merely

* This curve may or may not belong to the given system. 
the first order as hitherto, are found to be

$$
\begin{aligned}
& \left(Y^{\prime} X Y\right)=\frac{1}{2} F_{q} t^{2}+\frac{1}{3} M_{q} t^{3}+\cdots, \\
& \left(Z^{\prime} Z Y\right)=\frac{1}{2} G_{p} t^{2}+\frac{1}{3} N_{p} t^{3}+\cdots, \\
& -\left(Z^{\prime} Y Z\right)=q t+\frac{1}{2}\left\{p G_{p}+q F_{p}+2 q G_{q}\right\} t^{2}+\frac{1}{2}\left\{q\left(F_{p} G_{q}-F_{q} G_{p}\right)\right. \\
& \left.-G_{1}+q N_{q}+\frac{2}{3} p N_{p}+\frac{1}{3} q M_{p}\right\} t^{3}+\cdots, \\
& -\left(Y^{\prime} Y Z\right)=p t+\frac{1}{2}\left\{p G_{q}+q F_{q}+2 p F_{p}\right\} t^{2}+\frac{1}{2}\left\{p\left(F_{p} G_{q}-F_{q} G_{p}\right)\right. \\
& \text { where } \\
& \left.-F_{1}+p M_{p}+\frac{2}{3} q M_{q}+\frac{1}{3} p N_{q}\right\} t^{3}+\cdots, \\
& F_{1} \equiv F_{x}+p F_{y}+q F_{z}, \quad G_{1} \equiv G_{x}+p G_{y}+q G_{z} .
\end{aligned}
$$

Expanding the condition for a normal congruence, we find that there are no terms independent of $t$, the coefficient $\Omega_{1}$ of $t$ vanishes identically, the coefficient $\Omega_{2}$ of $t^{2}$ gives

$$
\left(1+q^{2}\right) F_{q}-\left(1+p^{2}\right) G_{p}+p q\left(F_{p}-G_{q}\right)+2(p G-q F)=0,
$$

and the coefficient $\Omega_{3}$ of $t^{3}$ gives

$$
\begin{aligned}
\left(1+q^{2}\right) M_{q}-(1 & \left.+p^{2}\right) N_{p}+p q\left(M_{p}-N_{q}\right)+3\left(p G-q F^{\prime}\right)\left(F_{p}+G_{q}\right) \\
& +3 p\left(G_{x}+p G_{y}+q G_{z}\right)-3 q\left(F_{x}+p F_{y}+q F_{z}\right)=0 .
\end{aligned}
$$

By means of $(23)$ and $\left(22^{\prime}\right)$ the equation $\left(24^{\prime}\right)$ may be written in the more convenient form

$$
\left(1+q^{2}\right) F_{1 q}-\left(1+p^{2}\right) G_{1 p}+p q\left(F_{1 p}-G_{1 q}\right)+3\left(p F_{1}-q G_{1}\right)=0 .
$$

In order that a quadruply infinite system

$$
y^{\prime \prime}=F\left(x, y, z, y^{\prime}, z^{\prime}\right), \quad z^{\prime \prime}=G\left(x, y, z, y^{\prime}, z^{\prime}\right)
$$

shall have the property that the $\infty^{2}$ curves through an arbitrary point form a normal congruence, it is necessary that the functions $F(x, y, z, p, q)$ and $G(x, y, z, p, q)$ fulfill the conditions (23) and (24).

As stated above, the coefficient of the first power of $t$ vanishes identically; in fact, if we think of the point as a small sphere, the curves passing through it, for any system (1), may be considered as meeting the sphere twice orthogonally. Equation (23) is the condition for approximation of the second degree; while (24) and (23) are the conditions for approximation of the third degree.

It is easily verified that all velocity systems

$$
y^{\prime \prime}=\left(\psi-y^{\prime} \phi\right)\left(1+y^{\prime 2}+z^{\prime 2}\right), \quad z^{\prime \prime}=\left(\chi-z^{\prime} \phi\right)\left(1+y^{\prime 2}+z^{\prime 2}\right),
$$


satisfy equation (23). Substituting in (24), however, we find a restriction, which reduces to

$$
\psi_{z}-\chi_{y}+p\left(\chi_{x}-\phi_{z}\right)+q\left(\phi_{y}-\psi_{x}\right)=0 .
$$

Since this is to hold identically, the result is a natural family. In this case we know that the congruences are actually of the normal type. Hence

THEOREM VI. The only velocity systems which have the property that the curves through an arbitrary point form a normal * congruence are the natural families.

By combining this with the first part of theorem V, we obtain the following

Theorem VII. If a quadruply infinite system is such that the congruences constructed by taking as base every straight line and every point of space are normal, then the same will be true when the base is any surface or any curve, and the system must be natural. $\dagger$

It is easily seen that conditions (23) and (24), expressing the approximate fulfillment of the condition for a normal congruence in the case of base points, are not of themselves sufficient to characterize natural families. In fact the general solution of these two partial equations for the determination of $F$ and $G$, one of the first and the other of the second order, would involve arbitrary functions of four arguments, and thus would include the natural type, which involves a function of only three arguments, as a very special case.

This question now arises: Can a system of $\infty^{4}$ curves, other than the known natural type, be such that the $\infty^{2}$ curves through an arbitrary point form a normal congruence? That the answer is in the affirmative we show by an example. The direct attack on the problem, according to the method hitherto pursued in this paper, would lead to great difficulties; it would require the discussion of the endless set of equations, of successively higher order, obtained by adjoining to (23) and (24) the conditions derived from the powers of $t$ beyond the third.

To obtain an appropriate example, we consider the systems whose equations are of the special form

$$
y^{\prime \prime}=F(p, q), \quad z^{\prime \prime}=G(p, q),
$$

the variables $x, y, z$ being absent. Excluding the case where $F$ and $G$ both vanish, such systems are not natural. They are characterized by the fact that they admit the group of all translations. It will therefore be sufficient to test the character of the curves through a single point, for instance, the origin.

For the functions appearing in (25), we observe, from $\left(22^{\prime}\right)$, that the derived functions $F_{1}$ and $G_{1}$ vanish and hence condition (24) is satisfied. Condition

* It is sufficient to demand the third degree of approximation.

$\dagger$ It is sufficient to demand the first approximation in the case of the straight lines, and the third in the case of the points. 
(23) may be fulfilled by taking either $F$ or $G$ at random and calculating the other function; this can easily be effected by quadratures. Thus all systems allowing the complete group of translations and such that the curves through an arbitrary point fulfill the normal condition up to terms of the third order may be obtained by quadratures.

It will be sufficient to consider the special case where $G$ is assumed to vanish, that is, where the curves are assumed to lie in planes perpendicular to the $x z$ plane. The systems are then found to be of the form

$$
y^{\prime \prime}=p^{2} f\left(\frac{1+q^{2}}{p^{2}}\right), \quad z^{\prime \prime}=0,
$$

where $f$ is an arbitrary function of one argument.

Consider now the particular system

$$
y^{\prime \prime}=p^{2}, \quad z^{\prime \prime}=0,
$$

included in this form. The integral curves are

$$
y=\log \frac{a_{1}}{b x+a}, \quad z=b x+b_{1},
$$

involving four arbitrary constants. The $\infty^{2}$ curves passing through the origin are

$$
y=\log \frac{a}{x+a}, \quad z=b x .
$$

The differential equations of this congruence are

$$
\frac{d x}{x}=\frac{d y}{e^{y}-1}=\frac{d z}{z}
$$

the corresponding total equation,

$$
x d x+\left(e^{y}-1\right) d y+z d z=0,
$$

is actually integrable, giving as the $\infty^{1}$ orthogonal surfaces

$$
x^{2}+z^{2}+2\left(e^{y}-y\right)=\text { constant. }
$$

Our example (27), which evidently is not of the natural type, enables us to state

Thborem VIII. A quadruply infinite system may have the property that the curves through an arbitrary point form a normal congruence, without. necessarily being a natural system.

Not all systems of the form (26) have the required property. For consider the particular system

$$
y^{\prime \prime}=\frac{1}{2}\left(1+q^{2}\right), \quad z^{\prime \prime}=0,
$$

whose integral curves are the parabolas

$$
y=\left(1+b^{2}\right) x^{2}+a x+a_{1}, \quad z=b x+b_{1} .
$$


The congruence through the origin is

$$
y=\left(1+b^{2}\right) x^{2}+a x, \quad z=b x,
$$

with the differential equations

$$
\frac{d x}{x}=\frac{d y}{y+x^{2}+z^{2}}=\frac{d z}{z} .
$$

Since the corresponding total equation

$$
x d x+z d z+\left(y+x^{2}+z^{2}\right) d y=0
$$

is not integrable, the congruence is not normal.

This example shows that a system may be such that the curves through an arbitrary point form a congruence which is normal to the third degree of approximation without being exactly normal.

\section{§5. The Family of SURfaCes Related to an aRbitraRy System.}

Given an arbitrary quadruply infinite system of curves

$$
y^{\prime \prime}=F(x, y, z, p, q), \quad z^{\prime \prime}=G(x, y, z, p, q),
$$

where $p$ and $q$ stand for $y^{\prime}$ and $z^{\prime}$ respectively, it is evident that in general there will exist no surface such that the $\infty^{2}$ curves of the system which meet the surface orthogonally form a normal congruence. If, however, it is merely required that the $\infty^{2}$ curves shall be of the normal character to the first approximation, that is, shall be orthogonal to some consecutive surface, then solutions will always exist.

The requisite condition on the surface

$$
z=f(x, y)
$$

is furnished by equation $\left(10^{\prime}\right)$. In the present discussion $F$ and $G$ are considered as the given functions and $f^{\prime}$ is to be found. Rearranging the equation in terms of second derivatives of $f$, we write it

$$
B_{0}+B_{1} f_{x x}+B_{2} f_{x y}+B_{3 .} f_{y, y}=0,
$$

where the coefficients are functions of $x, y, z, p, q$, determined by $F$ and $G$ as follows:

$$
\begin{aligned}
q B_{0} & \equiv\left(1+p^{2}+q^{2}\right)\left(q F_{x}-F_{z}-p G_{.}+G_{y}\right), \\
B_{1} & \equiv\left(1+p^{2}+q^{2}\right)\left(p q F_{p}+q^{2} F_{q}-p^{2} G_{p}-p q G_{q}\right)+2\left(p^{2}+q^{2}\right)\left(p G-q F^{\prime}\right), \\
B_{2 .} & \equiv\left(1+p^{2}+q^{2}\right)\left(2 p G_{p}+q G_{q}-q F_{p}\right)+2 p q F-2\left(q^{2}+2 p^{2}\right) G, \\
B_{3} & \equiv 2_{p} G_{r}-\left(1+p^{2}+q^{2}\right) G_{p} . \\
\text { Truns. Am. Yath. Soc. 10 } &
\end{aligned}
$$


Here $p$ and $q$ take the place of $P$ and $Q$ respectively in the discussion of $\S 2$, so that we must substitute

$$
p=\frac{f_{y}^{\prime}}{f_{x}}, \quad q=-\frac{1}{f_{x}} .
$$

After the substitution (30), (32), the coefficients $B_{i}$ become known functions of $x, y, f, f_{x}, f_{y}$. Denoting these new functions by $\bar{B}_{i}$, we have

THEOREM IX. With any quadruply infinite system of curves (29), there is associated a certain family of surfaces, namely, the solutions of the Monge partial differential equation of the second order

$$
\bar{B}_{0}+\bar{B}_{1} f_{x x}+\bar{B}_{2} f_{x y}+\bar{B}_{3} f_{y y}=0,
$$

where the coefficients are found from $F$ and $G$ by differentiations and eliminations. The $\infty^{2}$ curves of the given system which are orthogonal to any surface of this family are also orthogonal to some consecutive surface, that is, form an approximate normal congruence.

In case the system is of the natural type, equation (33) is of course an identity in $f$. In fact the set of equations obtained by putting the four coefficients $\bar{B}_{i}$, or the corresponding coefficients $B_{i}$, separately equal to zero is equivalent to the set (11) whose solution yielded the natural systems in the discussion of $\S 2$.

When will (33) reduce to an equation of the first order? The conditions for this are

$$
B_{1}=B_{2}=B_{3}=0 \text {, }
$$

which, in the notation of equations (11), are seen to be equivalent to

$$
C_{1}=C_{2}=C_{3}=0 \text {. }
$$

The system must therefore be of the velocity type

$$
y^{\prime \prime}=(\psi-p \phi)\left(1+p^{2}+q^{2}\right), \quad z^{\prime \prime}=(\chi-q \phi)\left(1+p^{2}+q^{2}\right) .
$$

In this case, $(33)$ reduces to

$$
\left(\psi_{z}-\chi_{y}\right) f_{x}+\left(\chi_{x}-\phi_{z}\right) f_{y}-\left(\phi_{y}-\psi_{x}\right)=0 .
$$

The integral surfaces are generated by the curves (characteristics)

$$
\frac{d x}{\psi_{z}-\chi_{y}}=\frac{d y}{\chi_{c}-\phi_{z}^{-}}=\frac{d z}{\phi_{y}-\psi_{x}} .
$$

These are the curl lines connected with the vector field* $\phi, \psi, \chi$.

Theorem X. For a general system of cirrves the associated equation (33) is actually of the second order, the only exceptions arising as follows: for

\footnotetext{
* This represents the force which generates the velocity system.
} 
a natural system, the equation holds identically; for any other velocity system the equation is merely of the first order. In the first case the associated family of surfaces is thus larger, and in the second smaller, than usual.

This deficiency refers to proper surfaces. We have already seen that, in the velocity type, an arbitrary curve employed as base gives rise to a congruence of approximately normal character. Thus, in addition to the proper surfaces (34), all curves may be regarded as limiting cases belonging to our family (33). In general we may with LIE replace the concept surface by the more general concept of union (Elementverein), that is, manifold of $\infty^{2}$ surface elements in united position. We might in fact have started out from this point of view and thereby should have avoided the separate discussion of curves and points. However this would have required the introduction of appropriate systems of homogeneous coördinates for differential surface elements.

One of our former results may be stated in the form: If the equation (33) is satisfied by all quadrics, or merely by the paraboloids

$$
z=a+b x+c y+a_{1} x^{2}+b_{1} x y+c_{1} y^{2},
$$

it must hold identically, so that the corresponding system must be natural. We now discuss the analogous question for spheres.

The $\infty^{4}$ spheres of space may be defined by the set of partial differential equations

$$
\frac{f_{x x}}{1+f_{x}^{2}}=\frac{f_{x y}}{f_{x} f_{y}}=\frac{f_{y y}}{1+f_{y}^{2}} \text {. }
$$

If then the associated family of surfaces is to include the spheres, equation (33) must be satisfied in consequence of (36). Eliminating. $f_{x x}$ and $f_{y y}$ by means of (36), and making use of (32), we find that (33) reduces to

$$
p B_{0}+\left\{\left(1+q^{2}\right) B_{1}+p B_{2}+\left(p^{2}+q^{2}\right) B_{3}\right\} f_{x y}=0 .
$$

This must be true for all values of $f_{x y}$; hence

that is,

$$
B_{0}=0, \quad\left(1+q^{2}\right) B_{1}+p B_{2}+\left(p^{2}+q^{2}\right) B_{3}=0,
$$

$$
q F_{x}-F_{z}-p G_{x}+G_{y}=0,
$$

$$
\left(1+q^{2}\right) F_{q}-\left(1+p^{2}\right) G_{p}+p q\left(F_{p}-G_{q}\right)+2(p G-q F)=0 .
$$

The latter is recognized as the condition that all points shall give rise to approximately (of first degree) normal congruences. The other equation, (37), is easily seen to be the condition that (33) shall be satisfied by all planes.

If for each point and for each plane as base, the congruence of orthogonal curves is approximately of normal character, then the same is true for an arbitrary sphere. The systems of this sort are defined by equations (37), (38).*

* The result involves arbitrary functions of four arguments, bence is more general than the natural type. 
It is easily seen that the only velocity systems satisfying condition (37) are those of the natural type. Making use of theorem V, we thus have

Theorem XI. If a system of curves is such that the $\infty^{2}$ curves orthogonal to any straight line or to any plane are orthogonal to some neighboring surface then the system must be natural.

If for an arbitrary curve as base the congruence of orthogonal curves is (exactly) normal, then the same must be true for any point as base. For the curve may be made to shrink up to the point. Hence, by combining the converse result of theorem IV with theorem VI, we have

Theоnem XII. Natural systems are characterized by the requirement that the curves of the system which are orthogonal to an arbitrarily selected curve (inside or outside the system) form a normal congruence.

It is even sufficient to take as base curves merely the $\infty^{6}$ circles of space.

Columbia University, New York. 